\title{
Enfermeiros intensivistas e não intensivistas e suas condutas de saúde diante do estresse profissional
}

\author{
Intensivist and non-intensivist nurses and their health conducts in the face of professional stress \\ Las Enfermeras Intensivistas Y No Intensivistas Y Sus Conductas Sanitarias Ante El Estrés \\ Profesional
}

Recebido: 14/04/2021 | Revisado: 25/04/2021 | Aceito: 29/04/2021 | Publicado: 13/05/2021

Francisco Ariclene Oliveira

ORCID: https://orcid.org/0000-0002-0290-4797 Centro Universitário Fametro, Brasil

E-mail: ariclene.oliveira@professor.unifametro.edu.br

Luís Adriano Freitas Oliveira

ORCID: https://orcid.org/0000-0002-8248-1404 Centro Universitário Fametro, Brasil

E-mail: adrianojs03@gmail.com

Denizielle de Jesus Moreira Moura

ORCID: https://orcid.org/0000-0003-3727-2416 Centro Universitário Fametro, Brasil

E-mail: deniziellemoreira@gmail.com

Arisa Nara Saldanha de Almeida

ORCID: https://orcid.org/0000-0002-8637-5174 Centro Universitário Fametro, Brasil E-mail: arisanara@gmail.com

Lucélia Rodrigues Afonso

ORCID: https://orcid.org/0000-0002-8370-4008 Universidade Estadual do Ceará - UECE, Brasil

E-mail: luceliarodriguess@yahoo.com.br

Cláudia Patrícia da Silva Ribeiro Menezes

ORCID: https://orcid.org/0000-0002-0559-9302

Universidade Estadual do Ceará, Brasil

E-mail:claudia_ribeiro6@hotmail.com

Lívia Lopes Custodio

ORCID: https://orcid.org/0000-0001-9610-7379

Universidade Estadual do Ceará, Brasil

E-mail: liviacustodio@yahoo.com.br

Samila Sousa Vasconcelos

ORCID: https://orcid.org/0000-0002-1509-1887

Centro Universitário Inta, Brasil

E-mail: samilasousa@hotmail.com

Débora Cristina Couto Oliveira Costa

ORCID: https://orcid.org/0000-0002-9402-0613

Universidade Estadual do Ceará, Brasil

E-mail: deboraccoc@gmail.com

Janaina dos Santos Mendes

ORCID: https://orcid.org/0000-0002-6200-6294

Universidade Estadual do Ceará, Brasil

E-mail: janainasmendes22@gmail.com

Macedônia Pinto dos Santos

ORCID: https://orcid.org/0000-0003-1582-2720

Universidade Estadual do Ceará, Brasil

E-mail: macedonia.pinto@gmail.com

Ilvana Lima Verde Gomes

ORCID: https://orcid.org/0000-0002-1861-5121

Universidade Estadual do Ceará, Brasil

E-mail: ilverde@gmail.com

\section{Resumo}

O presente estudo teve como objetivo verificar os fatores de estresse profissional e as condutas de saúde dos enfermeiros intensivista e não intensivista de uma unidade terciária de saúde no município de Fortaleza. Estudo transversal, descritivo, realizado de agosto a dezembro de 2019. A amostra constituiu-se de 83 enfermeiros intensivistas e não intensivistas convocados através da amostra por compatibilidade. Recorreu-se de um inquérito organizado com questões fechadas sobre o perfil socioeconômico, condutas de saúde, os indícios de estresse 
profissional e presença de fatores estressores. Os resultados mostraram que $64,7 \%$ dos intensivistas faziam uso de substâncias psicoativas, entre os não intensivistas esse percentual é apenas de 27,3\%. Os dados revelam que todos os profissionais da UTI (100\%) disseram apresentar algum sintoma de estresse. A presença de sintomas de estresse entre os participantes que não atuam na UTI alcançou 93,9\%. Para os enfermeiros não intensivistas, os principais fatores desencadeadores de estresse no lugar onde são desenvolvidas suas funções são: 'condição no ambiente insatisfatório e ausência de insumos' (56,1\%) e 'ausência de reconhecimento profissional' (53\%). Verificou-se que $82,3 \%$ dos participantes da UTI apontaram a 'insatisfação com a remuneração’ como primeiro agente estressor, acompanhado de 'sobrecarga de atividades' (70,6\%) e 'processo de trabalho desgastante' (58,8\%). Acredita-se que esse estudo possa ser favorável, afim de que os gerentes ponderem sobre os elementos influenciadores para o bem-estar dos profissionais enfermeiros, cooperando no sentido de implementar práticas que recuperem o estado laboral, favorecendo a autoconfiança no trabalho.

Palavras-chave: Estresse ocupacional; Enfermeiros; Unidades de terapia intensiva; Cuidados intensivos; Comportamento saudável.

\begin{abstract}
The present study aimed to verify the professional stress factors and the health behaviors of intensive care and nonintensive care nurses from a tertiary health unit in the city of Fortaleza. Cross-sectional, descriptive study, carried out from August to December 2019. The sample consisted of 83 intensive and non-intensive nurses called through the sample for compatibility. An organized survey was used with closed questions about the socioeconomic profile, health behaviors, signs of professional stress and the presence of stressors. The results showed that $64.7 \%$ of intensivists used psychoactive substances, among non-intensivists this percentage is only $27.3 \%$. The data reveal that all ICU professionals $(100 \%)$ said they had some symptom of stress. The presence of stress symptoms among participants who do not work in the ICU reached 93.9\%. For non-intensive care nurses, the main factors that trigger stress in the place where their functions are developed are: 'unsatisfactory environment and absence of inputs' (56.1\%) and 'absence of professional recognition' (53\%). It was found that $82.3 \%$ of ICU participants pointed to 'dissatisfaction with remuneration' as the first stressor, accompanied by 'overload of activities' (70.6\%) and 'exhausting work process' $(58.8 \%)$. It is believed that this study may be favorable, in order for managers to consider the elements that influence the well-being of professional nurses, cooperating in order to implement practices that recover the work state, favoring self-confidence at work.
\end{abstract}

Keywords: Occupational stress; Nurses; Intensive care units; Intensive care; Healthy behavior.

\title{
Resumen
}

El presente estudio tuvo como objetivo verificar los factores de estrés profesional y los comportamientos de salud de enfermeras de cuidados intensivos y no intensivos de una unidad terciaria de salud de la ciudad de Fortaleza. Estudio descriptivo transversal, realizado de agosto a diciembre de 2019. La muestra estuvo conformada por 83 enfermeros intensivos y no intensivos convocados a través de la muestra por compatibilidad. Se utilizó una encuesta organizada con preguntas cerradas sobre el perfil socioeconómico, conductas de salud, signos de estrés profesional y presencia de estresores. Los resultados mostraron que el $64,7 \%$ de los intensivistas usaban sustancias psicoactivas, entre los no intensivistas este porcentaje es solo del 27,3\%. Los datos revelan que todos los profesionales de UCI (100\%) dijeron tener algún síntoma de estrés. La presencia de síntomas de estrés entre los participantes que no laboran en la UCI alcanzó el 93,9\%. Para las enfermeras de cuidados no intensivos, los principales factores que desencadenan el estrés en el lugar donde se desarrollan sus funciones son: 'ambiente insatisfactorio y ausencia de insumos' $(56,1 \%)$ y 'ausencia de reconocimiento profesional' (53\%). Se constató que el 82,3\% de los participantes en UCI señalaron la 'insatisfacción con la remuneración' como primer factor estresante, acompañada de 'sobrecarga de actividades' $(70,6 \%)$ y 'proceso de trabajo agotador' $(58,8 \%)$. Se cree que este estudio puede ser favorable, para que los gerentes consideren los elementos que influyen en el bienestar de los profesionales de enfermería, colaborando para implementar prácticas que recuperen el estado laboral, favoreciendo la autoconfianza en el trabajo.

Palabras clave: Estrés laboral; Enfermeras; Unidades de cuidados intensivos; Cuidados intensivos; Comportamiento.

\section{Introdução}

O estresse ocupacional é definido pela International Labour Organization (ILO) como uma resposta física e emocional causada por um desequilíbrio entre as atividades laborais, tensões expositivas da labuta e habilidades do indivíduo para lidar com situações estressoras (ILO, 2016). As atividades laborais na saúde apresentam características complexas, contínua e atinge mudanças socioeconômicas e tecnológicas. Nesta perspectiva, as mudanças repentinas no exercício profissional permeiam o estresse dos profissionais e, à vista disso, levando o trabalhador de saúde a enfermidade. Portanto, o estresse ocupacional é uma condição patológica resultante de um ambiente laboral deletério, correlacionado aos elementos corporativos, às circunstâncias laborais e à condição das junções entre pessoas no ambiente de trabalho (Carvalho, et al., 2020). 
Desta maneira, é crucial que a pessoa admita o seu grau de estresse, assim como, seus causadores no local de trabalho e, principalmente ter a sapiência de que o estresse ocupacional é promotor para o desencadeamento de algumas patologias dos sistemas cardiovasculares, musculoesqueléticos, gastrointestinais e psicológicos (Ribeiro, et al., 2018; Prado, 2016).

Dentre as categorias profissionais que estão expostas aos fatores de estresse, dá-se destaque aos trabalhadores da enfermagem, em especial os enfermeiros intensivista. Salienta-se que a ocorrência exacerbada de estresse ocupacional entre esses profissionais, está associada ao local, complicações de vínculos humanos e de emprego, independência trabalhista, alta qualidade de competências e habilidades, planejamento de recursos humanos e materiais. Para tanto, o estresse ocupacional causa risco a saúde dos profissionais, além de corroborar com impactos negativos nas atividades laborais, como: redução do desempenho, baixo moral, rotatividade dos funcionários, absenteísmo e desentendimentos (Lindholm, 2006; Schmidt, et al., 2006).

Ademais, o ambiente hospitalar é considerado como um fator estressor, e que apresenta condições de insalubridade e periculosidade comparado a outros tipos de ambientes de saúde, como consultórios, unidade de atenção primária a saúde e/ou home care. Contudo, no ambiente hospitalar, a Unidade de Terapia Intensiva (UTI) é considera o local completo e propulsor para a exposição dos fatores de estresse ocupacional. Neste sentido, a UTI apresenta características singulares e corriqueiras nas rotinas laborais, sendo estas: as situações de sofrimento entre paciente e familiares, exposição a ruídos sonoro, risco eminente de contaminação, dentro outros fatores (Brochado \& Ribas, 2018).

No que concerne a identificação dos fatores de estresse ocupacional atrelada ao contexto organizacional, destacam-se: o grande número de doentes, o encargo excessivo de trabalho, a redução dos trabalhadores de enfermagem e de recursos insuficientes e/ou materiais de consumo para o atendimento de qualidade ao paciente (Simões, Otani \& Siqueira Junior, 2015).

Considera-se que os trabalhadores da enfermagem, em especial os enfermeiros que atuam em unidades intensivas, estão expostos a uma gama de atividades desgastantes e que proporcionam ao desenvolvimento do estresse ocupacional por conviverem diariamente com o sofrimento alheio, manutenção empática com os pacientes e com a equipe multiprofissional, submeter-se as fragilidades políticas que restringem os recursos humanos e/ou materiais de consumo, dentre outros.

Objetivou-se verificar os fatores de estresse profissional e as condutas de saúde dos enfermeiros intensivista e não intensivista de uma unidade terciária de saúde no município de Fortaleza.

\section{Metodologia}

Concerne de um estudo descritivo, transversal, com abordagem quantitativa, orientado por meio das observações do Strengthening the Reporting of Observational Studies in Epidemiology - STROBE (Malta, et al., 2010). O estudo ocorreu em uma unidade hospitalar do município Fortaleza pertencente ao Sistema Único de Saúde (SUS), no intervalo de agosto a dezembro de 2019.

Para definição da amostra, tomou-se como referência o número de profissionais enfermeiros que atuavam na UTI geral (Unidades A e B). A seleção dos participantes das demais unidades para composição da amostra, deu-se a partir da técnica de pareamento por setor de atuação na UTI, assim obteve-se 83 participantes, sendo 17 enfermeiros da UTI e 66 enfermeiros que atuam em outras unidades (clínicas médica e cirúrgica), tendo a proporção de 1:3. Os participantes elegíveis para a pesquisa foram recrutados por meio da técnica de amostragem por conveniência (Yamamoto \& Landim, 2013).

Os critérios de inclusão foram: Ter a prática profissional ligada à assistência e ter aproximadamente seis meses de experiência profissional na instituição. Os critérios de exclusão compreenderam em: estar afastado por licença médica, licença maternidade e férias no período em que os dados foram coletados.

A ponto de reunir os dados, foi utilizado um questionário autoaplicável e já validado, com 11 questões fechadas de múltipla escolha relacionadas aos fatores de estresse ocupacional e comportamentos de saúde, que contemplou a temática da 
pesquisa (Scholze, et al., 2017; Souza, Silva \& Costa, 2018; Llapa-Rodriguez, et al., 2018).

O instrumento de coleta de dados foi agrupado em 6 blocos classificados em: A - Especificações da instrução dos componentes do estudo; B - Episódios estressores alusivo ao local de trabalho; C - Utilização de elementos psicoativos (tabaco, cafeína, medicamentos, álcool e outras drogas); D - Condutas de bem-estar praticados por colaboradores de saúde; E Indícios de estresse por parte dos profissionais; F - Reconhecimento das condições estressoras fundamentais nos trabalhadores participantes. Para aplicação do questionário, o tempo estimado foi de cerca de 15 minutos.

Os dados alcançados foram digitados e preparados, aplicados em planilhas eletrônicas do programa Microsoft ${ }^{\circ}$ Office Excel@ 2016. Depois da tabulação dos dados, aplicou-se a análise estatística descritiva por meio do Software STATA, computando-se as frequências absolutas e relativas.

Em consonância com aspectos éticos legais de estudo envolvendo seres humanos, esta pesquisa obteve aprovação do serviço para sua execução e foi devidamente aprovado pelo Comitê de Ética e Pesquisa com seres humanos, sob parecer de $\mathrm{n}^{\circ}$. 2.903.371.

\section{Resultados}

O estudo elenca quatro tabelas que expressam o perfil socioeconômico, o uso de elementos psicoativos e condutas de saúde, a presença de sintomas e fatores de estresse, referidos por 17 enfermeiros intensivistas e 66 não intensivistas, que trabalham na assistência à saúde diretamente, de um hospital terciário do município de Fortaleza, apresentado por meio de características especificações da instrução dos componentes do estudo, conforme segue a tabela abaixo. 
Tabela 1 - Características pessoais, escolares e trabalhistas dos participantes. Fortaleza (CE), 2021.

\begin{tabular}{|c|c|c|c|}
\hline \multirow{2}{*}{ Variáveis } & \multirow{2}{*}{ Total } & \multirow{2}{*}{$\frac{\text { Não Intensivista }}{\text { n }(\%)}$} & \multirow{2}{*}{$\frac{\text { Intensivista }}{n(\%)}$} \\
\hline & & & \\
\hline \multicolumn{4}{|l|}{ Sexo } \\
\hline Masculino & 9 & $4(6,1)$ & $5(29,4)$ \\
\hline Feminino & 74 & $62(93,9)$ & $12(70,6)$ \\
\hline \multicolumn{4}{|l|}{ Idade } \\
\hline 18 a 49 anos & 66 & $52(78,8)$ & $14(82,4)$ \\
\hline 50 a 65 & 16 & $13(17,7)$ & $3(17,6)$ \\
\hline 65 ou mais anos & 1 & $1(1,5)$ & $0(0,0)$ \\
\hline \multicolumn{4}{|l|}{ Escolaridade } \\
\hline Graduação & 11 & $6(9,1)$ & $5(29,4)$ \\
\hline Especialização & 64 & $54(81,8)$ & $10(58,8)$ \\
\hline Mestrado & 5 & $3(4,5)$ & $2(11,7)$ \\
\hline Doutorado & 3 & $3(4,5)$ & $0(0,0)$ \\
\hline \multicolumn{4}{|l|}{ Tempo de formado } \\
\hline$<1$ ano & 3 & $3(4,5)$ & $0(0,0)$ \\
\hline 1 a 5 anos & 26 & $23(34,8)$ & $3(17,6)$ \\
\hline 6 a 10 anos & 21 & $16(24,2)$ & $5(29,4)$ \\
\hline 11 a 15 anos & 5 & $2(3,0)$ & $3(17,6)$ \\
\hline 16 a 19 anos & 8 & $5(7,6)$ & $3(17,6)$ \\
\hline$<20$ anos & 19 & $16(24,2)$ & $3(17,6)$ \\
\hline Não respondeu & 1 & $1(1,5)$ & - \\
\hline \multicolumn{4}{|l|}{ Tempo de serviço na instituição } \\
\hline$<1$ ano & 17 & $16(24,2)$ & $1(5,9)$ \\
\hline 1 a 5 anos & 21 & $17(25,8)$ & $4(23,5)$ \\
\hline 6 a 10 anos & 21 & $15(22,7)$ & $6(35,3)$ \\
\hline 11 a 15 anos & 6 & $3(4,5)$ & $3(17,6)$ \\
\hline 16 a 19 anos & 3 & $2(3,0)$ & $1(5,9)$ \\
\hline$<20$ anos & 14 & $12(18,2)$ & $2(11,8)$ \\
\hline Não respondeu & 1 & $1(1,5)$ & - \\
\hline \multicolumn{4}{|l|}{ Possui outro emprego } \\
\hline Sim & 37 & $26(39,4)$ & $11(64,7)$ \\
\hline Não & 44 & $38(57,6)$ & $6(35,3)$ \\
\hline Não respondeu & 2 & $3(3,0)$ & - \\
\hline \multicolumn{4}{|c|}{ Vínculo trabalhista com a instituição } \\
\hline Cooperada(o) & 48 & $37(56,1)$ & $11(64,7)$ \\
\hline Estatutária(o) & 25 & $19(28,8)$ & $6(35,3)$ \\
\hline Celetista & 8 & $8(12,1)$ & $0(0,0)$ \\
\hline Residente & 1 & $1(1,5)$ & $0(0,0)$ \\
\hline Não respondeu & 1 & $1(1,5)$ & - \\
\hline
\end{tabular}

Fonte: Autores (2021).

Observou-se que as mulheres compõem a força de trabalho mais importante do hospital, representando $93,9 \%$ dos profissionais de saúde não intensivistas e 70,6\% intensivistas, apresentado uma característica forte da profissão. No que corresponde a idade, 52,9\% dos intensivistas têm idade entre 26 e 33 anos, assim apresentando prevalência de 36,4\% entre os não intensivistas. Quanto à escolaridade, verificou-se que os enfermeiros que não atuam na UTI apresentaram maiores níveis de formação, haja vista que $81,8 \%$ possuíam especialização e 4,5\% já tinham concluído doutorado. Entre os intensivistas, 29,4\% relataram possuir apenas a graduação, 58,8\% eram especialistas e nenhum participantes com doutorado. Constatou-se que $39,3 \%$ dos profissionais que atuam nas unidades clínicas possuem um tempo inferior à de 5 anos de formados e, entre os enfermeiros intensivistas com formação abaixo de 5 anos corresponderam a 17,6\%, logo, a prevalência de $29,4 \%$ foi de 6 a 10 anos de formado. 
Averiguou-se que 25,8\% dos profissionais não intensivistas possuem menos de 5 anos de atuação na unidade de saúde e 24,2\% variando entre 6 e 12 meses. Contudo, 18,2\% revelam trabalhar no serviço há mais de vinte anos. Para tanto, 35,3\% dos intensivistas atuam na instituição e apenas 11,8\% tinham mais de 20 anos de atuação no hospital. Em relação ao vínculo trabalhista no hospital, percebeu-se que 56,1\% são cooperados e, que não intensivistas e 64,7\% dos enfermeiros da UTI são cooperados. Verificou-se que $57,6 \%$ dos enfermeiros não intensivista só possuíam a referida instituição como único meio de trabalho e, entre os intensivistas $64,7 \%$ possuíam outro emprego.

Conforme apresentado na Tabela 2, 64,7\% dos profissionais que trabalham na UTI fazem uso de substâncias psicoativas, entre os não intensivistas esse percentual é apenas de 27,3\%. Entre profissionais da UTI, a cafeína (47,1\%) e o álcool $(17,6 \%)$ foram as substâncias mais prevalentes. O uso de cigarro não foi reportado por nenhum dos participantes.

Tabela 2 - Uso de substância psicoativas e comportamentos de saúde referidos pelos participantes. Fortaleza (CE), 2021.

\begin{tabular}{|c|c|c|c|}
\hline \multirow{2}{*}{ Variáveis } & \multirow{2}{*}{ Total } & \multirow{2}{*}{$\frac{\text { Não Intensivista }}{\text { n }(\%)}$} & \multirow{2}{*}{$\frac{\text { Intensivista }}{\mathrm{n}(\%)}$} \\
\hline & & & \\
\hline \multicolumn{4}{|l|}{ Uso de substância psicoativa } \\
\hline Não & 54 & $48(72,7)$ & $6(35,3)$ \\
\hline Sim & 29 & $18(27,3)$ & $11(64,7)$ \\
\hline \multicolumn{4}{|l|}{ Tipo de substância* } \\
\hline Cafeína & 21 & $13(19,7)$ & $8(47,1)$ \\
\hline Álcool & 5 & $2(3,0)$ & $3(17,6)$ \\
\hline Medicamento & 8 & $8(12,1)$ & $0(0,0)$ \\
\hline \multicolumn{4}{|l|}{ Comportamento de saúde } \\
\hline Não & 19 & $16(24,2)$ & $3(17,6)$ \\
\hline Sim & 64 & $50(75,8)$ & $14(82,4)$ \\
\hline \multicolumn{4}{|c|}{ Tipo de comportamento de saúde } \\
\hline Atividade física & 38 & $31(47,0)$ & $7(41,2)$ \\
\hline Alimentação saudável & 29 & $18(27,3)$ & $11(64,7)$ \\
\hline Tocar ou ouvir música & 22 & $22(33,3)$ & $0(0,0)$ \\
\hline Uso de medicamento & 4 & $4(6,1)$ & $0(0,0)$ \\
\hline Terapia & 1 & $0(0,0)$ & $1(5,9)$ \\
\hline Atividades religiosas & 14 & $2(3,0)$ & $12(70,6)$ \\
\hline
\end{tabular}

$*$ Tabaco $=0(0,0 \%)$. Fonte: Autores (2021).

Verificou-se que $82,4 \%$ dos participantes que atuam na UTI praticavam algum dos tipos de comportamento de saúde. A adoção de comportamentos de saúde pelos não intensivistas foi reportada por $75,9 \%$. Dentre as atividades de comportamentos de saúde mais reportadas pelos profissionais intensivistas, identificou-se: 'atividades religiosas' - 70,6\%, 'alimentação saudável' - 64,75\% e 'atividade física'- 41,2\%. Para os profissionais não intensivistas, os comportamentos de saúde mais citados foram: a prática de 'atividade física' com 47,0\% e 'tocar/ouvir música' com 33,3\%.

Apresenta-se a seguir, a Tabela 3 reunindo algumas características das variáveis referentes à manifestação de sintomas de estresse relatadas pelos participantes. Os dados apontam que 100,0\% dos enfermeiros da UTI apresentaram sintoma de estresse. A presença de sintomas de estresse entre os participantes que não atuam na UTI alcançou 93,9\%. 
Tabela 3 - Existência de manifestações de estresse entre os enfermeiros intensivistas e não intensivista. Fortaleza (CE), 2021.

\begin{tabular}{|c|c|c|c|}
\hline Variáveis & Total & $\frac{\text { Não Intensivista }}{\text { n }(\%)}$ & $\frac{\text { Intensivista }}{\mathrm{n}(\%)}$ \\
\hline \multicolumn{4}{|l|}{ Sintomas de estresse } \\
\hline Sim & 79 & $62(93,9)$ & $17(100,0)$ \\
\hline Não & 4 & $4(6,1)$ & $0(0,0)$ \\
\hline \multicolumn{4}{|l|}{ Estratificação de sintomas de estresse } \\
\hline 1 a 5 sintomas & 45 & $38(57,6)$ & $7(41,2)$ \\
\hline 6 a 10 sintomas & 26 & $19(28,8)$ & $7(41,2)$ \\
\hline 11 a 14 sintomas & 7 & $4(6,1)$ & $3(17,6)$ \\
\hline 15 a 20 sintomas & 5 & $5(7,6)$ & $0(0,0)$ \\
\hline \multicolumn{4}{|l|}{ Tipo de sinais/sintomas ${ }^{*}$} \\
\hline Tensão muscular & 51 & $36(54,5)$ & $15(88,2)$ \\
\hline Desgaste físico & 45 & $33(50,0)$ & $12(70,6)$ \\
\hline Insônia & 39 & $35(53,0)$ & $4(23,5)$ \\
\hline Irritabilidade excessiva & 33 & $25(37,9)$ & $8(47,1)$ \\
\hline Mudança de apetite & 32 & $22(33,3)$ & $8(47,1)$ \\
\hline Problemas de memória & 30 & $17(25,8)$ & $6(35,3)$ \\
\hline Perda do senso de humor & 24 & $14(21,2)$ & $10(58,8)$ \\
\hline Agitação & 23 & $22(33,3)$ & $10(58,8)$ \\
\hline Cansaço constante e excessivo & 23 & $21(31,8)$ & $2(11,8)$ \\
\hline Alterações gástricas & 22 & $18(27,3)$ & $4(23,5)$ \\
\hline Formigamento das extremidades & 22 & $15(22,7)$ & $7(41,2)$ \\
\hline Alterações dermatológicas & 22 & $13(19,7)$ & $9(52,9)$ \\
\hline Apatia, depressão ou raiva prolongada & 20 & $13(19,7)$ & $7(51,2)$ \\
\hline Hipersensibilidade emotiva & 20 & $13(19,7)$ & $7(41,2)$ \\
\hline Boca seca & 19 & $15(22,7)$ & $4(23,5)$ \\
\hline Taquicardia & 19 & $15(22,7)$ & $4(23,5)$ \\
\hline Angustia e/ou ansiedade e/ou medo diariamente & 15 & $15(22,7)$ & $0(0,0)$ \\
\hline \multicolumn{4}{|l|}{ Mal-estar generalizado (sem causa específica) } \\
\hline Diminuição da libido & 13 & $9(13,6)$ & $4(23,5)$ \\
\hline Hiperventilação & 10 & $7(10,6)$ & $3(17,6)$ \\
\hline Náusea & 8 & $4(6,1)$ & $4(23,5)$ \\
\hline Pesadelos frequentes & 5 & $5(7,6)$ & $0(0,0)$ \\
\hline \multirow[t]{2}{*}{ Hipertensão } & 4 & $4(6,1)$ & $0(0,0)$ \\
\hline & 1 & $1(1,5)$ & $0(0,0)$ \\
\hline
\end{tabular}

*Ideação suicida $=0$ (0,0). Fonte: Autores (2021).

Verificou-se que 57,6\% dos enfermeiros não intensivistas apresentavam de 1 a 5 manifestações de estresse, consecutivos os que referem apresentar de 6 a 10 ocorrências, corresponde a 28,8\%. Entre os intensivistas, 41,2\% referiram expressar de 1 a 5 sinais/sintomas de estresse, percentual idêntico aos que relataram manifestar entre 6 a 10 sinais/sintomas. Dentre as manifestações com maior prevalência de estresse entre os enfermeiros de enfermarias clínicas, observou-se a 'tensão muscular' (54,5\%), 'insônia' (53,0\%) e 'desgaste físico' (50,0\%). Os sinais/sintomas mais prevalentes entre os intensivistas foram: 'tensão muscular' (88,2\%), ‘desgaste físico' (70,6\%), ‘mudança de apetite' e 'alteração de humor’ (ambos com 58,8\%), 'alterações dermatológicas' (52,9\%) e 'apatia, depressão ou raiva prolongada' (51,2\%).

A Tabela 4 os enfermeiros não intensivistas, apontaram os principais fatores desencadeadores de estresse no ambiente de trabalho são: 'estrutura ambiental deficiente e falta de materiais' (56,1\%) e 'ausência de reconhecimento profissional' $(53,0 \%)$. Verificou-se que $82,3 \%$ dos participantes da UTI apontaram a ‘insatisfação com a remuneração’ como principal fator de estresse, seguido de 'sobrecarga de atividades' (70,6\%) e 'processo de trabalho desgastante' (58,8\%). Observou-se que fatores como: 'dimensionamento de pessoal', 'trabalho noturno', 'tomada de decisões de conflitos' e 'falta de autonomia profissional' foram apontados como fatores estressores, mas não foram mencionados pelos enfermeiros intensivistas. 
Tabela 4 - Causas de estresse mencionados por enfermeiros não intensivistas e intensivistas. Fortaleza (CE), 2021.

\begin{tabular}{|c|c|c|c|}
\hline Variáveis & Total & $\begin{array}{c}\text { Não Intensivista } \\
\mathrm{n}(\%) \\
\end{array}$ & $\frac{\text { Intensivista }}{\mathrm{n}(\%)}$ \\
\hline Fator de estresse ocupacional & & & \\
\hline Não & 2 & $2(3,0)$ & $0(0,0)$ \\
\hline Sim & 81 & $64(97,0)$ & $17(100,0)$ \\
\hline Tipos de fatores de estresse & & & \\
\hline Insatisfação com a remuneração & 46 & $32(48,5)$ & $14(82,3)$ \\
\hline Estrutura ambiental deficiente e falta de materiais & 44 & $37(56,1)$ & $7(41,2)$ \\
\hline Ausência de reconhecimento profissional & & & \\
\hline Processo de trabalho desgastante & 42 & $35(53,0)$ & $7(41,2)$ \\
\hline Sobrecarga das atividades & $\begin{array}{l}39 \\
38\end{array}$ & $\begin{array}{l}29(43,9) \\
26(39,4)\end{array}$ & $\begin{array}{l}10(58,8) \\
12(70,6)\end{array}$ \\
\hline Carga horária & 36 & $28(42,4)$ & $8(47,1)$ \\
\hline Relacionamento interpessoal com os demais profissionais & 27 & $19(28,8)$ & $8(47,1)$ \\
\hline Falta de autonomia profissional & & & \\
\hline Dimensionamento de pessoal & 16 & $16(24,2)$ & $0(0,0)$ \\
\hline Trabalho noturno & 16 & $16(24,2)$ & $0(0,0)$ \\
\hline Exposição constante a riscos & 14 & $14(21,1)$ & $0(0,0)$ \\
\hline Trabalho repetitivo & 15 & $8(12,1)$ & $7(41,2)$ \\
\hline Tomada de decisões de conflitos & 13 & $9(13,6)$ & $4(23,5)$ \\
\hline $\begin{array}{l}\text { 1 omada de decisoes de conflitos } \\
\text { Lidar com a morte }\end{array}$ & 11 & $11(16,7)$ & $0(0,0)$ \\
\hline & 8 & $4(6,1)$ & $4(23,5)$ \\
\hline $\begin{array}{l}\text { Conflito entre o trabalho e a família } \\
\text { Acidente de trabalho }\end{array}$ & 5 & $2(3,0)$ & $3(17,6)$ \\
\hline Acidente de trabalho & 4 & $1(1,3)$ & $3(17,6)$ \\
\hline
\end{tabular}

Fonte: Autores (2021).

\section{Discussão}

No Brasil, cerca de $85,1 \%$ do perfil feminino dos profissionais da enfermagem, atuam na UTI e unidades clínicas. No tocante à idade, observou-se que na faixa etária entre 26 e 33 anos em ambos os estratos populacionais pesquisados foram prevalentes. Esses resultados apontam que a profissão de enfermagem é predominantemente liderada por mulheres, sendo uma tendência histórica da profissão. Nas últimas décadas, observou-se que 14,4\% dos homens atuam enfermagem (Machado, et al., 2016).

A maioria dos profissionais das enfermarias clínicas eram especialistas, além de profissionais com formação de mestrado e doutorado, o que comprova que as atividades ocupacionais, tem solicitado, atuação mais qualificada dos profissionais. Os profissionais de saúde especialistas na área, facilitam a interação e integração com o processo de trabalho, promovem soluções imediatas e corretas, conduzem com presteza a equipe dos profissionais e, visam a diminuição de ameaças a vida e integridade do doente (Freitas, et al., 2015).

Referente ao período de formação e de serviço na unidade de saúde, concluiu-se que a maior parte dos intensivistas do estudo tinha entre 6 a 10 anos de formado, cenário que destoa entre os enfermeiros de unidades clínicas em que a maioria tem menos de 5 anos de formado, período semelhante ao tempo de vínculo com a instituição. Os dados sobre inserção no mercado de trabalho mostram que o emprego para enfermeiros vem crescendo no país (IPEA, 2020).

No entanto, constatou-se que perto de um quinto dos profissionais não intensivistas relataram ter mais de duas décadas de formação, número expressivo dos trabalhadores com tempo maior que vinte anos vinculado ao serviço, sendo estes que exibiam vínculo empregatício estatucional de trabalho. Assim é apontado pela literatura que os profissionais se adaptam ao local de serviço à medida que o tempo de trabalho vai se prolongando, dessa maneira desfavorecendo os fatores estressores. Em contrapartida, ter um tempo prolongado na ocupação é capaz de conduzir a banalização do seu processo de trabalho. A inquietação com a retirada sem substituição de profissionais qualificados, caracterizando-se de modo a representar um cenário 
de carências dos Enfermeiros. Essa realidade tem relação com o envelhecimento dos trabalhadores, com consecutiva disposição a desatenção da ocupação trabalhista devido à aposentadoria ou adoecimento (Buchan, O`May \& Dussault, 2013).

Evidenciou-se a hegemonia de pessoas com pouco tempo de trabalho na unidade de saúde, haja vista que a grande maioria dos intensivistas e não intensivistas relatara possuir tempo inferior a dez anos de serviço no hospital. Este fato tem relação com o percentual de profissionais que prestam serviço com vínculo de trabalho na modalidade de cooperado. Diante do panorama de fragilidade do processo trabalhista, os resultados salientam perdas para a saúde do profissional e para a instituição por conta do revezamento de pessoal e à evasão de capital racional que influenciam continuadamente na qualidade do atendimento prestado (Oliveira \& Teixeira, 2016).

Observou-se, que um quinto de profissionais recém-formados, tinham entre 6 e 12 meses de serviço na unidade de saúde. A fragilidade da força de trabalho na enfermagem ocorre devido à escassez de concursos públicos como forma de estruturação do quadro de funcionários permanente das instituições de saúde no Brasil, estão evidenciados nos resultados obtidos no estudo (Dias et al., 2019).

No que concerne ao tempo laboral, os profissionais de enfermagem iniciantes, são propícios a apresentar níveis elevados de estresse em comparação aos trabalhadores que já exercem suas atividades laborais há um certo tempo. Dessa maneira, percebe-se que quanto mais tempo de trabalho, menos sintomas de estresse o profissional irá apresentar, devido ao fato do enfermeiro possuir maior segurança técnica que adquiriu no decorrer dos anos, e domínio sobre as circunstâncias que aparecem em sua prática habitual de serviço (Guido, et al., 2011).

Certificou-se que grande parte dos enfermeiros não intensivistas trabalham apenas em uma unidade de saúde. Essa veracidade, evidência o desajuste em se harmonizar dois empregos, uma vez que, no ambiente hospitalar onde os profissionais de enfermagem representam a maior força laboral, o trabalho torna-se repetidamente complexo, além de tudo estão sujeitos a uma hierarquia inflexível para cumprir horários, rotinas, condutas e regimentos (Machado, et al., 2016).

Contudo, entre os intensivistas, observou-se prevalência para a jornada dupla de empregos e, encontra-se atrelada ao complemento da renda familiar. Ressalta-se que esse revezamento da escala de trabalho (dia-noite) altera o estágio de repouso, causando desgaste no profissional, podendo atingir a sua capacidade, a condição física, os aspectos psicológicos, o seu contentamento, o convívio familiar e a vida social (Cechin, et al., 2014). Pontua-se que a dupla jornada de trabalho é aplicada como agente de enfermidades físicas e/ou mentais em profissionais de saúde, além de contribuir episódios de ausência no trabalho, acidentes no trabalho, falhas no preparo de medicamentos, esgotamento, encargo em atividades laborais e afastamento das diversões (Correa \& Andrade, 2020).

Identificou-se que os profissionais pesquisados fazem uso de substâncias psicoativas, sendo maioria entre os intensivistas. Dentre estas substâncias, a cafeína, o álcool e os medicamentos são os mais frequentes. Esse achado é corroborado a de um outro estudo realizado com 123 profissionais de saúde que trabalhavam em um Hospital Geral do Estado de Alagoas, no qual verificou que 37,4\% dos trabalhadores usavam medicações psicoativas. O estudo ainda aponta que os profissionais que faziam uso dessas substâncias alegavam circunstâncias precárias no trabalho, carga horária extensa, inúmeras privações sono e repouso, e estresse como motivo primordial para o uso dessas drogas (Maciel et al., 2017). Em estudo que avaliou uso de substância entre enfermeiros, constatou-se que esses profissionais apresentam 30 a 100 vezes mais chances que a população em geral de ficarem com dependência química, além de exibirem um grande grau de síndrome de exaustão, se comparados com outros profissionais (Rocha \& David, 2015).

Verificou-se que o uso de cigarro não foi reportado por nenhum dos participantes, dado considerado bastante positivo, haja visto que esse resultado difere de um outro estudo que identificou a prevalência do tabagismo em profissionais de enfermagem, no qual identificou que dos 656 participantes, 10,2\% eram tabagistas (Ayoub \& Sousa, 2019).Acredita-se que esse resultado possa ser justificado pelo fato de esses profissionais apresentarem formação em saúde e, consequentemente, 
conhecem os males ocasionados pelo tabaco (Madewell, el al., 2017).

Acredita-se que o reconhecimento de condutas vigorosas de saúde, são práticas inexistentes nos hábitos assíduos na saúde dos profissionais, demandando dos gestores e das instituições a promoção de intervenção que fortaleçam a adoção de tais práticas. Salienta-se que a adoção de atitudes e estilo de vida benéficos por trabalhadores de saúde é primordial para a promoção qualidade de vida (Neall, Atherton \& Kyle, 2016).

Os dados da pesquisa revelam que todos os intensivistas pesquisados referiram apresentar algum fator de estresse relacionados ao ambiente de trabalho. A existência de agentes estressores entre os integrantes que não atuam na UTI alcançou 97,0\%. Esses resultados sinalizam que os profissionais de enfermagem que atuam na assistência direta ao paciente clínico ou grave, apresentam percentuais de prevalência da presença de estresse, evidenciando a necessidade premente de ações que vise a redução/mitigação desses elementos estressores. Os profissionais de enfermagem de uma UTIN de um hospital público do Espírito revelaram que 56,7\% dos participantes apresentaram sinais de estresse (Mello, Reis \& Ramos, 2018).

Os desfechos aduziram que grande parte dos enfermeiros não intensivistas apresentavam de 1 a 5 sinais/sintomas de estresse, seguido de $41,2 \%$ dos intensivistas que referiram expressar de 1 a 5 sinais/sintomas. Também foram expressivos os percentuais de profissionais de enfermarias clínicas e intensivistas que referiram apresentar de 6 a 10 manifestações. Dentre os sinais/sintomas mais prevalentes de estresse entre os enfermeiros de enfermarias clínicas, observou-se a 'tensão muscular', 'insônia' e 'desgaste físico'. Os sinais/sintomas mais prevalentes entre os intensivistas foram: 'tensão muscular', 'desgaste físico', 'mudança de apetite' e 'alteração de humor', 'alterações dermatológicas' e 'apatia, depressão ou raiva prolongada'. Um estudo recente sobre sintomas de estresse, realizado com profissionais que atuam em UTI, "indicou predominância dos seguintes sintomas: "sensação de desgaste físico constante", indicado por $70 \%$ da amostra; "cansaço excessivo", apontado por 63,3\% dos participantes; "cansaço constante" e "tensão muscular", ambos, atingindo o percentual de 56,7\% da amostra" (Mello, Reis \& Ramos, 2018).

As manifestações estressoras pactuam três etapas: primeiramente uma etapa referindo-se a uma resposta de proteção ou de aviso e que apresentam sintomatologia de taquicardia, palidez, a fadiga, a insônia e ausência de apetite; a segunda etapa é intitulada de resistência ou adaptativa, a qual a pessoa comprova os sintomas relacionados ao isolamento social, à inaptidão de desvincular-se do processo laboral, o nervosismo excessivo e à redução do apetite sexual; a terceira etapa, no que lhe diz respeito, se mostra como período de abatimento ou esgotamento. Na presente etapa, a pessoa revela distúrbios como hipertensão arterial, depressão, ansiedade, problemas sexuais e dermatológicos, como psoríase, vitiligo, urticárias e alergia, e também infarto ou mesmo morte súbita (Guido, et al., 2011).

De acordo com o apresentado, percebeu-se que os trabalhadores mencionaram apresentar sintomatologia de estresse categorizados nas três etapas de episódios dos sintomas. Essa conjuntura expõe verdadeiramente o processo laboral em que este está sobreposto por cenário estressor, os quais ocasionam o aparecimento de manifestações que devem interferir na saúde física e psicológica do trabalhador, assim, faz-se necessário implementar estratégias e ações que reduzam os elementos de estresse ocupacional, favorecendo um melhor ambiente laboral e, por conseguinte, menos adoecedor.

No que diz respeito ao reconhecimento de fatores de estresse, constatou-se que a maioria dos participantes não intensivistas apontam a 'condição local deficiente e ausência de insumos' e 'ausência de reconhecimento profissional' elementos desencadeadores de estresse no ambiente de trabalho. Para os intensivistas avaliados, a 'insatisfação com a remuneração' foi apontada como principal fator de estresse, seguido de 'sobrecarga de atividades' e 'processo de trabalho desgastante'. Os elementos estressores apontados nesta pesquisa estão em acordo com os resultados de uma revisão integrativa que avaliou estudos sobre estresse entre enfermeiro, a qual constatou que os principais elementos de estresse são: o fardo no trabalho, problemas envolvendo o paciente e procedimentos, organização estrutural da unidade de saúde (Azevedo, et al., 2017). 
De modo geral, observou-se que os fatores/situações de estresse reportadas pelos participantes dizem respeito ao próprio ambiente e ao seu processo de trabalho, a sua atuação no cargo, como também nas conexões com o grupo no interior da instituição, tornando-se apto de intervenção que favoreçam melhor condição de trabalho, consequentemente, repercutindo em melhor qualidade de vida.

$\mathrm{Na}$ execução do estudo, buscou-se manter todo o rigor metodológico inerente às pesquisas científicas. Contudo, reconhece-se as limitações metodológicas características do delineamento dos estudos transversais. Recomenda-se a realização de outras pesquisas que visem avaliar a associação de fatores estressores com a existência de sintomatologia de estresse ocupacional, colaborando para a produção de conhecimentos para a comunidade científica e sociedade.

\section{Conclusão}

Os fatores estressores são propícios aos profissionais de enfermagem, que são altamente vulneráveis a estes elementos, devidos a suas práticas diárias em locais insalubres como enfermarias clínicas ou mesmo Unidades de Terapia Intensiva UTI. Por causa disso, buscou-se através deste estudo constatar o comportamento de saúde e os elementos de estressores no ambiente laboral de enfermeiros intensivistas e não intensivistas de um hospital geral, em nível de atenção terciária, no município de Fortaleza-CE. Considera-se ter alcançado o objetivo proposto, levando em consideração os resultados obtidos sobre as condutas de saúde dos enfermeiros, das manifestações de estresses e dos seus elementos geradores correspondentes, condizente com o que se demonstrou durante a pesquisa.

Diante dos resultados apontados faz-se necessário que medidas preventivas sejam adotadas no âmbito coletivo e organizacional, afim de promover um ambiente laboral ávido e com meios materiais necessários a uma assistência de qualidade. Constata-se que para promover o bem-estar dos profissionais, torna-se extremamente necessário a adoção dessas medidas que possam contribuir para obter a satisfação do grupo, favorecendo a melhoria do desempenho, diminuindo, consequentemente, os encargos sócios-econômicos para o profissional e a instituição, ponderando a probabilidade de conter as ameaças de enfermidades e absenteísmo, além de favorecer a humanização do atendimento e a proteção do trabalhador.

\section{Referências}

Azevedo, V. G. B., Alves, S. M., Viana, L. V. M., Veras, K. C. B. B, Carvalho, M. L. \& Elias, C. M. V. M. V. (2017). Estresse ocupacional em profissionais de enfermagem que atuam em unidades de urgência e emergência. R. Interd, 10(4):112-124.

Ayoub, A. C. \& Sousa, M. G. (2019). Prevalência do tabagismo em profissionais de enfermagem de um hospital cardiovascular. Rev. Bras. Enferm, 72, 1, 173180.

Brochado C. \& Ribas, J. L. C. (2018). Estresse Da Equipe De Enfermagem Na UTI. Rev. Saúde Desenvol., $12(13), 44-57$.

Buchan, J., O`May, F. \& Dussault, G. (2013). Nursing workforce policy and the economic crisis: a global overview. J Adv Nurs, $45(3), 298-307$.

Carvalho, A. E. L., Frazão, I. S., Silva, D. M. R., Andrade, M, S., Vasconcelos, S. C. \& Aquino, J. M. (2020). Estresse dos profissionais de enfermagem atuantes no atendimento pré-hospitalar. Rev. Bras. Enferm, 73(2), 1-6.

Cechin, P., Freitas, H. M. B., Ilha, S., Martins, E. S. R. \& Souza, M. H. T. (2014). Alterações vivenciadas por profissionais de enfermagem que apresentam dupla jornada de trabalho. Rev enferm UFPE on line, 8(11), 3855-3861.

Correa, T. P. \& Andrade, R. F. C. (2020): "A saúde do servidor público em Xeque: um olhar para as causas do seu adoecimento", Revista Contribuciones a las Ciencias Sociales, https://www.eumed.net/rev/cccss/2020/06/saude-servidor-publico.html

Dias, M. O., Souza, N. V. D. de O., Penna, L. H. G., \& Gallasch, C. H. (2019). Percepção das lideranças de enfermagem sobre a luta contra a precarização das condições de trabalho. Revista da Escola de Enfermagem da USP, 53, e03492. https://doi.org/10.1590/s1980-220x2018025503492

Freitas, R. J. M., Lima, E. C. A., Vieira, É. S., Feitosa, R. M. M, Oliveira, G. Y. M. \& Andrade, L. V. (2015) Estresse do enfermeiro no setor de urgência e emergência. Rev. Enferm. UFPE on line. Recife, 9(Supl. 10):1476-83.

Guido, L. A., Linch, G. F. C., Pitthan, L. O. \& Umann, J. (2011). Estresse, coping e estado de saúde entre enfermeiros hospitalares. Rev Escol Enferm da USP, 45(6), 1434-1439. 
Instituto de Pesquisas Econômicas e Aplicadas (2020) (IPEA). Ranking traz ocupações com os maiores salários. Brasília: IPEA. $<$ https://www.ipea.gov.br/portal/index.php?option=com_content\&view=article\&id=18829>.

International Labour Organization. (2016) (ILO). WORKPLACE STRESS: A collective challenge. Turin.

Llapa-Rodriguez, E. O., Oliveira, J. K. A., Lopes Neto, D. L., Gois, C. F. L., Campos, M. P. A., Mattos, M. C. T. (2018). Estresse ocupacional em profissionais de enfermagem. Rev. Enferm. UERJ, 26, e19404.

Lindholm. M. (2006). Working conditions, psychosocial resources and work stress in nurses and physicians in chief managers' positions. J. Nurs. Manage. 14(2), 300-309.

Malta, M., Cardoso, L. O., Bastos, F. I., Magnanini, M. M. F. \& Da Silva, C. M. F. P. (2010). Iniciativa STROBE: subsídios para a comunicação de estudos observacionais. Rev. Saúde Pública, 44(3), 559-565.

Machado, M. H, Oliveira, E, Lemos, W, Lacerda, W. F, Filho, W. A, Wermelinger, M. et al. (2016). Mercado de trabalho da enfermagem: Aspectos Gerais. Enfermagem em Foco, 7, 35-53. <http://revista.cofen.gov.br/index.php/enfermagem/article/view/691>. https://doi.org/10.21675/2357707X.2016.v7.nESP.691.

Maciel, M. P. G. S, Santana, F. L, Martins, C. M. A, Costa, W. T, Fernandes, L. S. \& Lima, J. S. (2017). Uso de medicamentos psicoativos entre profissionais de saúde. Rev. enferm. UFPE on line, 11(7): 2881-2887.

Madewell, Z. J., Figueiredo, V. C., Harbertson, J., Pérez, R. L. \& Novotny, T. (2017). Exposure to smoking in soap operas and movies: smoking cessation and attempts to quit. Cad Saúde Pública. 33(3).

Mello, R. C. C., Reis, L. B. \& Ramos, F. P. (2018). Estresse em profissionais de enfermagem: importância da variável clima organizacional. Gerais, Rev. Interinst. Psicol. 11(2), 193-207.

Neall, R. A., Atherton, I. M. \& Kyle, R. G. (2016). Nurses' health-related behaviours: protocol for a quantitative systematic review of prevalence of tobacco smoking, physical activity, alcohol consumption and dietary habits. J Adv Nurs, 72(1):197-204.

Oliveira, A. F. C. \& Teixeira, E. R. (2016). Concepções sobre o uso da automedicação pelos trabalhadores de enfermagem em terapia intensiva oncológica. Rev. Enferm. UFPE online. 10(1):24-31. <http://www.revista.ufpe.br/>.

Prado, C. E. P. (2016). Estresse ocupacional: causas e consequências. Rev. Bras. Med. Trab., 14(3), 285-289.

Ribeiro, R. P., Marziale, M. H. P., Martins, J. T., Galdino, M. J. Q. \& Ribeiro, P. H. V. (2018). Estresse ocupacional entre trabalhadores de saúde de um hospital universitário. Rev. Gaúcha Enferm. 39, 1-6.

Robazzi, M. L. C. C., Mauro, M. Y. C., Secco, I. A. O., Dalri, R. C. M. B., Freitas, F. C. T., Terra, F. S., et al. (2012). Alterações na saúde decorrentes do excesso de trabalho entre trabalhadores da área de saúde. Rev Enferm UERJ. 20(4), 526-532.

Rocha, P. R. da, \& David, H. M. S. L. (2015). Padrão de consumo de álcool e outras drogas entre profission ais de saúde: retrato de alunos de cursos lato sensu de uma instituição pública. SMAD, Rev. Eletrônica Saúde Mental Álcool Drog, 11(1), 42-48. https://doi.org/10.11606/issn.1806-6976.v11i1p42-48

Simões, J. S., Otani, M. A. P. \& Siqueira Júnior, A. C. (2015). Estresse dos profissionais de enfermagem em uma unidade de urgência. REGRAD, UNIVEM/Marília-SP, 8(1), 75-95.

Schmidt, D. R. C., Dantas, R. A. S., Marziale, M. H. P. \& Laus, A. M. (2009). Estresse ocupacional entre profissionais de enfermagem do bloco cirúrgico. Texto Contexto Enferm, 18(2), 330-337.

Scholze, A. R., Martins, J. T. \& Galdino, M. Q. (2017). Ribeiro, R. P. Ambiente ocupacional e o consumo de substâncias psicoativas entre enfermeiros. Acta Paul. Enferm, 30(4), 404-411.

Souza, R. C., Silva, S. M. \& Costa, M. L. A. S. (2018). Estresse ocupacional no ambiente hospitalar: revisão das estratégias de enfrentamento dos trabalhadores de Enfermagem. Rev. Bras. Med. Trab., 16(4), 493-502.

Yamamoto, J. K. \& Landim, P. M. B. (2013). Geoestatística: conceitos e aplicações. Editora Oficina de Textos. 